HNO 2015 $63: 605$

DOI 10.1007/s00106-015-0051-4

Online publiziert: 29. August 2015

(c) Springer-Verlag Berlin Heidelberg 2015

\section{R. Knecht}

Klinik und Poliklinik für Hals-, Nasen und Ohrenheilkunde, Kopf-Hals-Chirurgie und Onkologie, Universitätsklinikum Hamburg-Eppendorf, Hamburg, Deutschland

\title{
Aktuelle Therapiekonzepte bei Kopf-Hals-Tumoren
}

\section{Highlights des Jahrestreffens der American Society for Clinical Oncology 2015}

Auch in diesem Jahr fand wie gewohnt im Juni der Jahreskongress der American Society of Clinical Oncology (ASCO) in Chicago statt. Entgegen der Tradition, dass eher nichtchirurgische Themen im Fokus stehen, wurde dieses Jahr eine Studie von Anil D'Cruz stark beachtet. Die Gruppe aus Mumbai zeigte, dass eine frühe elektive Neck-Dissection bei Frühstadien von Mundhöhlenkarzinomen mit einem verbesserten Gesamtüberleben von $12,5 \%$ und einem um $36 \%$ verminderten Sterberisiko einhergeht. Die Daten dieser großen, randomisierten Studie sind ebenfalls in diesem Jahr im New England Journal of Medicine veröffentlicht worden [1].

\section{》) Die wichtigsten Neuigkeiten werden für die tägliche Praxis erfasst}

In den Übersichtsartikeln werden weitere Themen, wie die differenzielle Wertigkeit der Platinchemotherapie und unterschiedliche Schemata bei der Radiochemotherapie behandelt. Ein weiterer Schwerpunkt ist die zunehmend wieder an Bedeutung gewinnende Immuntherapie sowie die Rolle des Biomarkers HPV (humanes Papillomvirus). Die wichtigsten Neuigkeiten werden für die tägliche Praxis erfasst und dabei in den Kontext der Studienresultate der vergangenen Jahre eingeordnet.

Im Gegensatz zum Vorjahr haben wir dieses Jahr insgesamt 4 Schwerpunkte für Sie herausgesucht. Zusätzlich zu der Primärtherapie und den Therapieansät- zen in der Rezidiv-/Metastasensituation haben wir einen gesamten Artikel der Immuntherapie gewidmet. Studien, die sich mit der Immunmodulation befassen, dominieren aktuell auch für Tumoren im Kopf-Hals-Bereich die Studienlandschaft. Trotz der vielversprechenden Immuncheckpointmodulation ist die Integration dieser Medikamente in die Standardtherapie noch Zukunftsmusik. Hierfür ist zunächst die Identifikation prädiktiver Marker für die eine Patientenselektion erforderlich. Ein weiteres Novum ist die Darstellung neuer Aspekte in der Therapie von Schilddrüsenkarzinomen.

Wir hoffen, Ihnen hiermit eine aktuelle und interessante Übersicht in der sich ständig wandelnden Forschungslandschaft im Bereich der Kopf-HalsOnkologie geben zu können.

Herzlichst

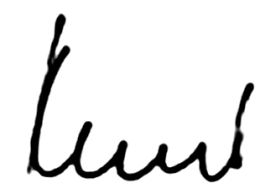

Ihr Rainald Knecht

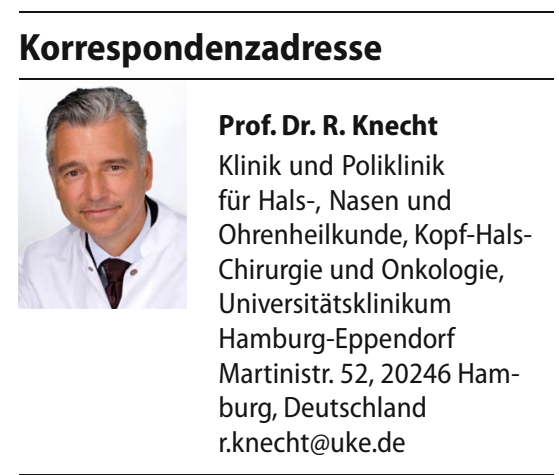

Interessenkonflikt. R. Knecht gibt an, dass kein Interessenkonflikt besteht.

\section{Literatur \\ 1. D'Cruz AK, Vaish $R$, Kapre $N$ et al (2015) Elective versus therapeutic neck dissection in node- negative oral cancer. N Engl J Med 373(6):521-529}

